\title{
¿Lectura pública electrónica? Evaluación del proyecto \\ E-Biblio en las bibliotecas públicas valencianas \\ (2015-2016)
}

\section{Electronic public reading? Evaluation of the EBiblio project in the Valencian public libraries (2015-2016)}

\author{
Miguel C. Muñoz Feliu \\ mimufe@,upvnet.upv.es
}

Universitat Politècnica de València. Dep. de Comunicació Audiovisual, Documentació i Història de l'Art (DCADHA)

Nuria Portillo Poblador

nportillo@eio.upv.es

\begin{abstract}
Universitat Politècnica de València. Dep. d'Estadística i Investigació
Operativa Aplicades i Qualitat
\end{abstract}

\section{Resumen}

Se estudia el conocimiento y grado de satisfacción con el programa E-Biblio de los usuarios de las bibliotecas públicas a partir del análisis de tres estudios realizados entre mayo de 2015 y junio de 2016. El alto grado de desconocimiento e insatisfacción tanto con los contenidos como con los formatos aconsejarían cambios en su difusión y prestaciones.

\section{Palabras clave}

Lectura pública, libro electrónico, E-Biblio, Comunitat Valenciana

\section{Abstract}

We study the knowledge and degree of satisfaction of public libraries users with the program E-Biblio from the analysis of three studies conducted between May 2015 and June 2016. The high degree of unknowledge and dissatisfaction, either contents or formats, advises changes in its distribution and performance.

Keywords

Public reading, Electronic book, E-Biblio, Comunitat Valenciana 
Recibido: 02/12/2016

Aceptado: 24/12/2016

DOI: http://dx.doi.org/10.5557/IIMEI7-N13-269283

Descripción propuesta: MUÑOZ FELIU, Miguel C.; PORTILLO POBLADOR, Nuria, 2016. ¿Lectura pública electrónica? Evaluación del proyecto E-Biblio en las bibliotecas públicas valencianas (2015-2016). Métodos de Información [en línea], 7(13), pp. 269-283.

\section{Introducción}

La Sociedad de la Información ha supuesto enormes cambios en todos los ámbitos de nuestra vida. También en el mundo del libro y de la lectura. Poco a poco, pero de una manera decidida, la lectura digital y el libro electrónico va ganando terreno frente al texto en papel. En España, de cada cien libros publicados 22 son ya en digital. Idéntico crecimiento se aprecia en el número de lectores en electrónico que en pocos años ha pasado del 6,5\% de 2011 al 17,7\% de 2015 (Observatorio de la Lectura y el Libro 2015, p. 1 y 65).

Las bibliotecas públicas han intentado adaptarse a estos cambios, una adaptación que supone cambios conceptuales muy profundos, entre ellos en el propio concepto de colección. Así, mientras que los libros en papel eran y son propiedad de la biblioteca tradicional, en el nuevo paradigma, la biblioteca dispone u ofrece acceso de los textos electrónicos, pero no como propietaria de los mismos. Ello conlleva limitaciones pero también nuevas posibilidades en servicios tradicionales muy demandados como el préstamo, antes ligados a un espacio físico y ahora posible mediante plataformas por Internet (Vicente García, 2013).

Numerosos países han establecido programas para el préstamo digital, casi siempre desde acciones coordinadas para todas las bibliotecas de la región o estado donde se pone en marcha (Mount, 2014). En España, el programa más importante llevado a cabo hasta ahora es el programa E-Biblio, iniciativa impulsada desde 2014 por el Ministerio de Educación, Cultura y Deporte y a 
la que se han sumado la mayoría de comunidades autónomas, entre ellas la Comunitat Valenciana. En sí, el sistema habilita un mecanismo de préstamo de contenidos legales por los que se paga a los editores a través de las administraciones, siendo el servicio gratuito para el ciudadano (Cuadrado Fernández y Fe Trillo, 2015).

A 31 de diciembre de 2015, la plataforma contaba con 4.098 títulos distintos y 240.157 licencias de uso (es decir, de posibles préstamos). Fueron usados durante ese año por 48.037 prestatarios, siendo la mayoría de las transacciones en verano. El número de préstamos ascendió durante 2015 a 246.115. Puede parecer una cifra alta. Sin embargo, es muy escasa si se compara con el préstamo presencial en las bibliotecas que superó en 2015 los 33 millones. Especialmente bajos son los resultados observados en las estadísticas oficiales en la Comunitat Valenciana, que figuraba muy por debajo de la media nacional en préstamos por cada 1000 habitantes (3.77 frente a 5.43) y en préstamos por cada 1000 inscritos (14.74 frente a 17.31) (Ministerio de Educación, Cultura y Deporte, 2016).

Existen estudios sobre el impacto y utilización del libro digital en otras regiones como Barcelona y Madrid (Domínguez Sánchez, 2011) o Andalucía (Gómez-Gómez, 2015). También hay comparaciones entre el perfil y conducta de los lectores digitales españoles y los de otras partes del mundo (Alonso Arévalo, Cordón García y Gómez Díaz, 2014).

Sin embargo, la Comunitat Valenciana no dispone de estudios fiables publicados, ni sobre la lectura digital en general, ni sobre el impacto del programa E-Biblio en particular. De ahí, la necesidad de este estudio que incidirá en dos variables fundamentales para que un programa de este tipo pueda tener éxito como es el conocimiento del mismo y la satisfacción de sus usuarios con él.

\section{Material y métodos}


Este estudio combina los resultados obtenidos en dos estudios, todos ellos con usuarios de bibliotecas públicas pertenecientes al Sistema Bibliotecario Valenciano.

El primero de ellos, realizado bajo nuestra dirección por Sara Pérez López fue llevado a cabo entre mayo y junio de 2015 fue llevado a cabo en tres bibliotecas de diferente tamaño y localización, con el fin de recoger un abanico lo más diverso posible de tipos de usuario (población urbana-rural, etc.) y de tipo de gestión de la propia entidad (municipal, pero también estatalautonómica). Las bibliotecas públicas seleccionadas de este primer estudio fueron la Biblioteca Pública Provincial de Valencia, ubicada en pleno centro de Valencia, la más concurrida de toda la Comunitat Valenciana, que es de titularidad estatal pero está gestionada por la comunidad autónóma; la Biblioteca Pública de Bétera, representante de una localidad mediana; y la Biblioteca Pública de Nàquera, en una localidad pequeña. El tipo de encuesta era presencial y los datos eran tomados por un entrevistador. Se realizaron a diversas horas y días para evitar sesgos por franjas horarias y obtener así una muestra representativa y en todos los casos se hacía a la salida de la biblioteca en cuestión para asegurar que el entrevistado fuera usuario real de la misma.

El segundo estudio se realizó en la Biblioteca Valenciana Nicolau Primitiu a los alumnos de un taller sobre el uso del libro electrónico en febrero de 2016. Los alumnos, provenientes tanto de Secundaria como de Formación de Adultos, público interesado por la lectura digital, completaban un formulario en papel antes del curso.

Finalmente, el tercer estudio se realizó en la Red de Bibliotecas Municipales del Ayuntamiento de Valencia, red que dispone de agencias de lectura o bibliotecas en la mayoría de barrios de la ciudad y en colaboración con la Sección de Bibliotecas del Ayuntamiento quien habilitó ordenadores ad boc para la realización de la encuesta online disponible en

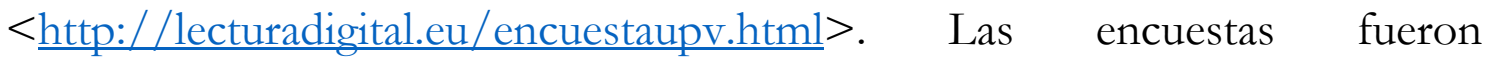
completadas por el público de manera voluntaria. Se realizaron entre mayo y junio de 2016.

En todos los casos se utilizó un formulario para valorar el conocimiento del sistema E-Biblio y también la satisfacción de los usuarios del sistema. .... 


\section{Resultados}

En este trabajo se recogen los resultados tres estudios. El número de cuestionarios recogidos en función del estudio se muestran en la Tabla 1.

\begin{tabular}{|l|l|l|l|}
\hline Estudio & Valor & Frecuencia & Porcentaje \\
\hline Bibliotecas públicas, a la salida & 1 & 207 & 0.5227 \\
\hline Taller uso libro electrónico & 2 & 147 & 0.3712 \\
\hline $\begin{array}{l}\text { Bibliotecas públicas, ordenadores } \\
\text { biblioteca. Cuestionario Castellano }\end{array}$ & 3 & 36 & 0.0909 \\
\hline $\begin{array}{l}\text { Bibliotecas públicas, ordenadores } \\
\text { biblioteca. Cuestionario Valenciano }\end{array}$ & 4 & 6 & 0.0152 \\
\hline
\end{tabular}

Tabla 1. Distribución de los cuestionarios según estudio.

Fuente: Elaboración propia

El perfil sociodemográfico y cultural de los encuestados es muy amplio y podemos considerarlo representativo de la sociedad valenciana actual. En cuanto a la edad de los participantes en el estudio, la edad mínima es de 12 años y la máxima de 79, siendo 32 años la edad mediana. El 23.2\% de los encuestados tiene entre 15 y 19 años. Respecto al sexo, el 38.5\% son hombres mientras que el $61.5 \%$ son mujeres.

\section{Nivel de Estudios}

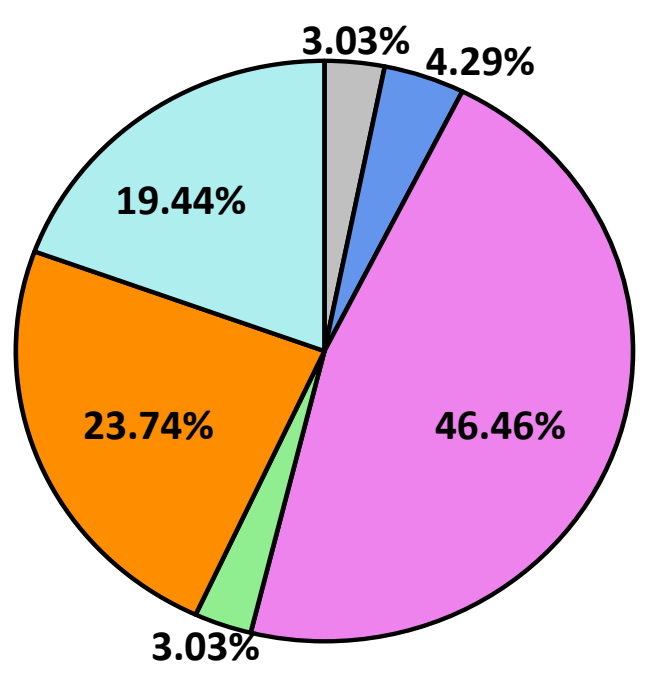

Nivel de Estudios

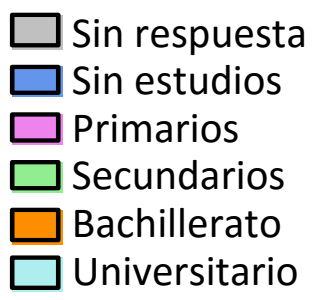

Gráfico 1. Nivel de Estudios. 
En cuanto a los estudios (Gráfico 1), el 46,46\% de los encuestados tienen estudios primarios, mientras que solamente el $19.44 \%$ tienen estudios universitarios.

En cuanto a la actividad principal que desarrollan (Gráfico 2), el 35.35\% son estudiantes, seguido de un $24 \%$ que trabajan por cuenta ajena mientras que el $15.91 \%$ busca trabajo y el $11.36 \%$ son jubilados.

\section{Principal Actividad}

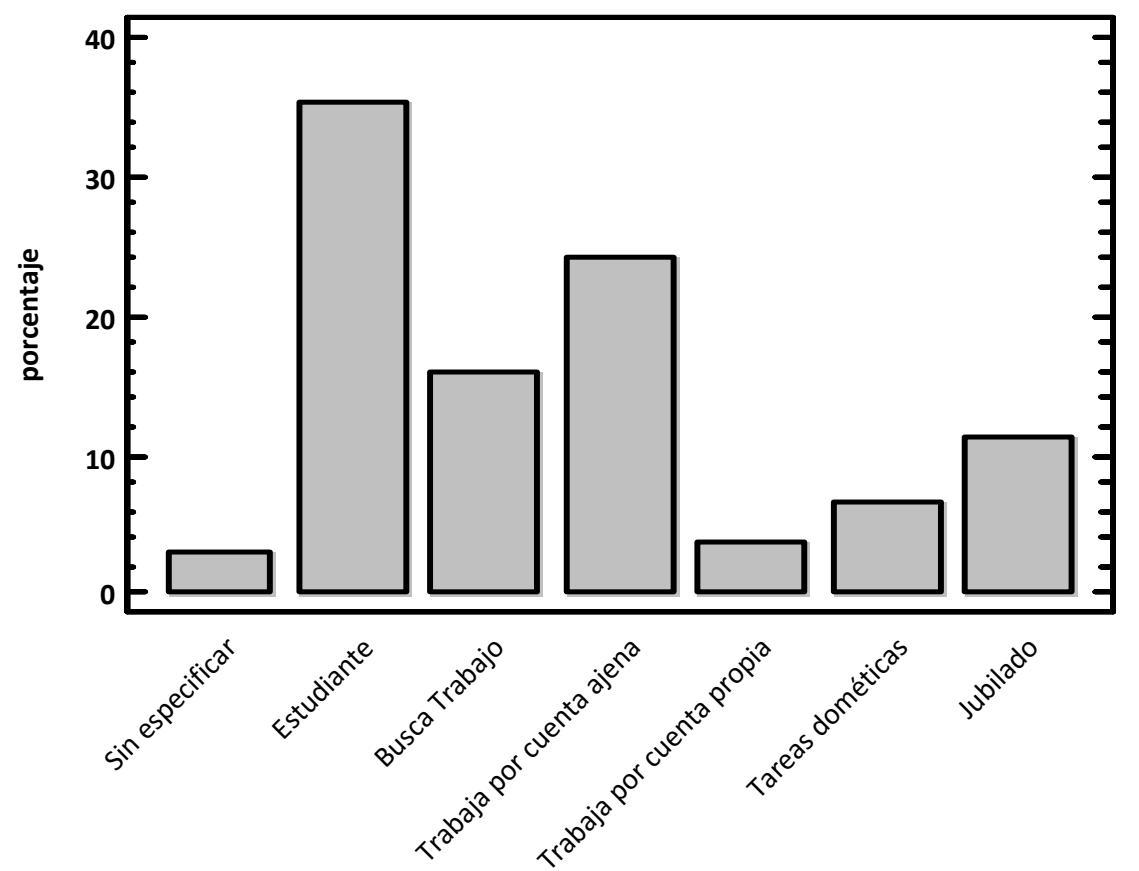

Gráfico 2. Principal Actividad.

Fuente: Elaboración propia

Respecto a la nacionalidad, la mayoría son españoles $(78.03 \%)$, pero también hay personas procedentes de otros países de la Unión Europea, China o América Latina.

A continuación, se analiza el perfil relacionado con las bibliotecas, sus hábitos de lectura y el servicio EBiblio.

El 74.68\% son lectores habituales y el resto no. En el Gráfico 3 se muestran la frecuencia con que visitan la biblioteca. Hemos de destacar que el 61.62\% 
visita la biblioteca al menos una vez por semana, con lo que se puede deducir que son usuarios habituales de la biblioteca.

\section{Vista Biblioteca}

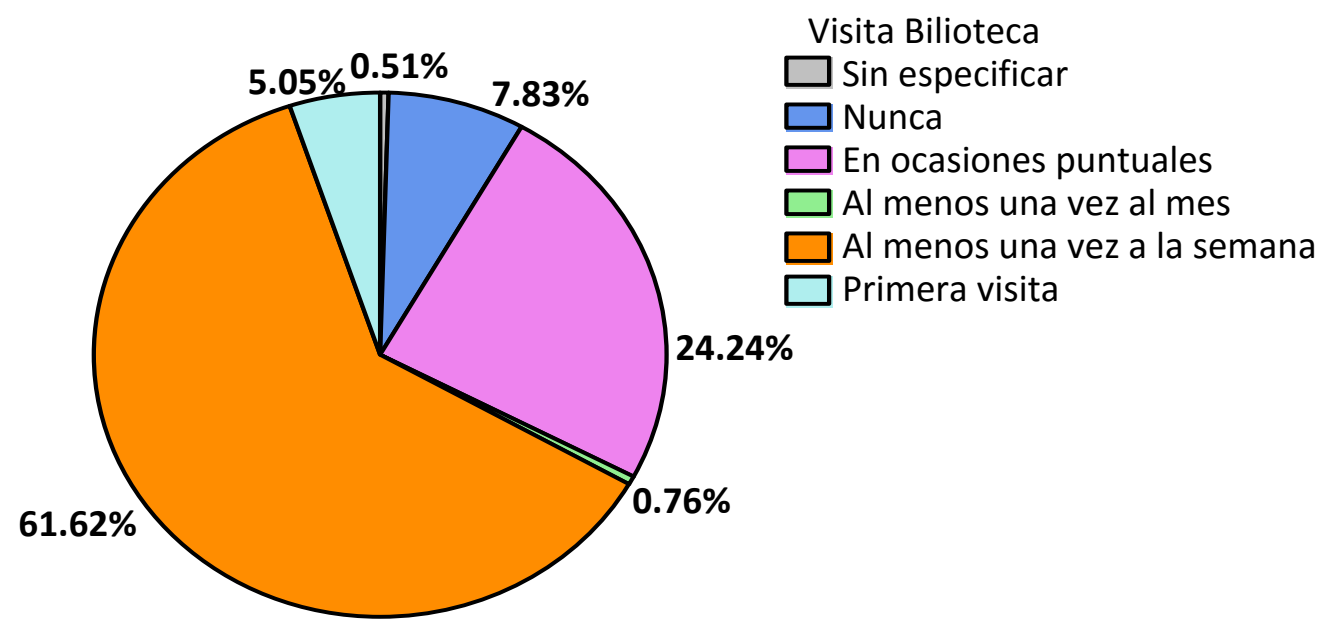

Gráfico 3. Visita Biblioteca. Fuente: Elaboración propia

Respecto al formato preferido para la lectura (Gráfico 4), el 76.01\% prefiere leer en papel y únicamente el $16.41 \%$ prefiere leer solamente en formato digital.

\section{Formato preferido de lectura}

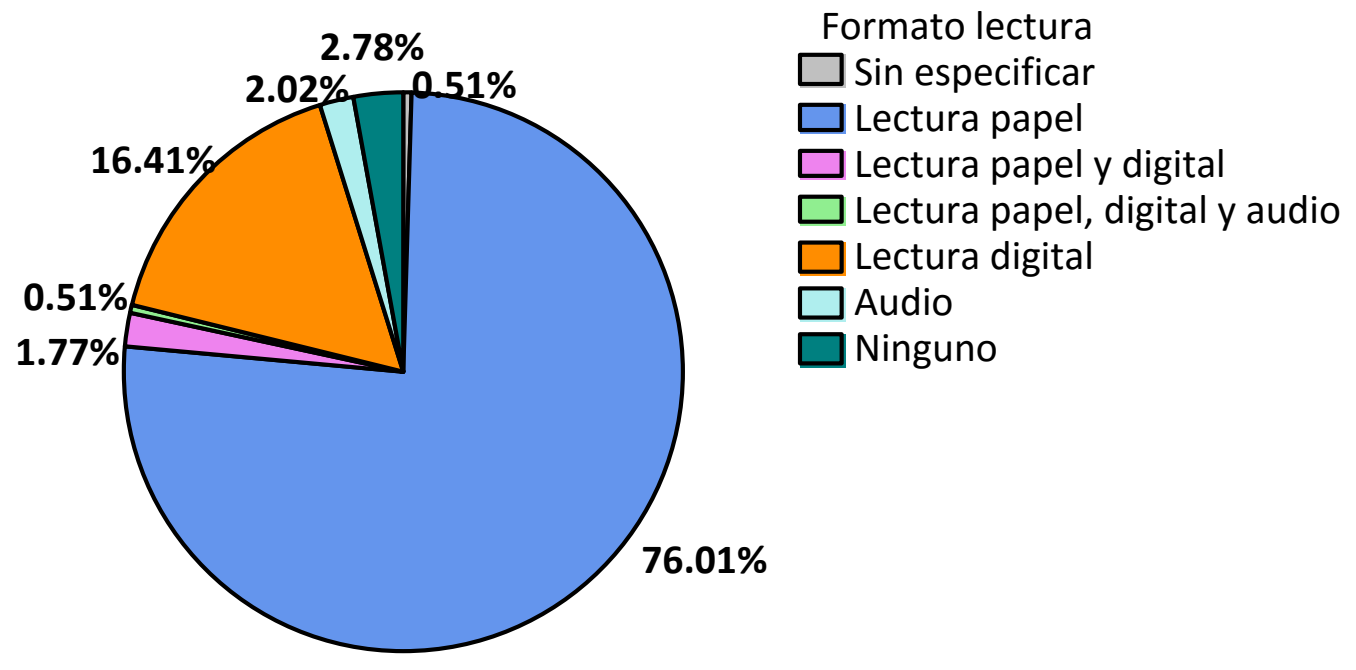

Gráfico 4. Formato preferido de lectura. Fuente: Elaboración propia 
Para los que utilizan la lectura digital, en la tabla 2 se indica el dispositivo electrónico que utiliza. Algunos usuarios utilizan distintos dispositivos. Así el $44.59 \%$ utiliza un libro electrónico no Kindle mientras que el 16.22\% utiliza Kindle.

Como el servicio EBiblio no se puede utilizar para los dispositivos Kindle, el $16.22 \%$ no podría utilizar el servicio con su dispositivo electrónico de lectura.

\begin{tabular}{|l|l|l|}
\hline Dispositivo Electrónico & Valor & Porcentaje \\
\hline Ordenador & 18 & 0.243243243 \\
\hline Movil/Tablet & 19 & 0.256756757 \\
\hline Libro electrónico no Kindle & 33 & 0.445945946 \\
\hline Kindle & 12 & 0.162162162 \\
\hline
\end{tabular}

Tabla 2. Dispositivo electrónico utilizado. Fuente: Elaboración propia

En cuanto a si conocen el servicio EBiblio, en el Gráfico 5 se observa que el $90.15 \%$ de los encuestados no conoce el servicio.

\section{Conocimiento servicio EBiblio}

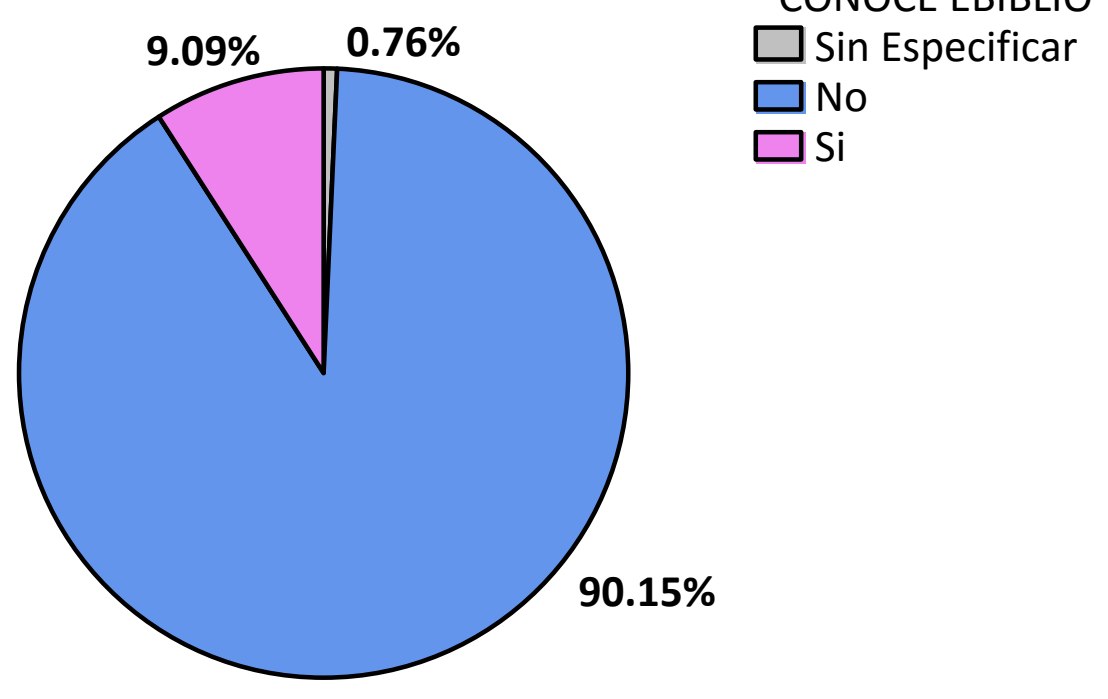

Gráfico 5. Conocimiento servicio EBiblio. Fuente: Elaboración propia 
Independientemente del estudio del que se trate, la falta de conocimiento del servicio EBiblio es generalizada. Se plantea una prueba de independencia para averiguar si el estudio y el conocimiento de la biblioteca son independientes. El resultado es afirmativo con un riesgo de primera especie del $0 \%$. En la Tabla 3 y en el Gráfico 6 se observa lo especificado en este párrafo.

\section{Conocimiento EBiblio}

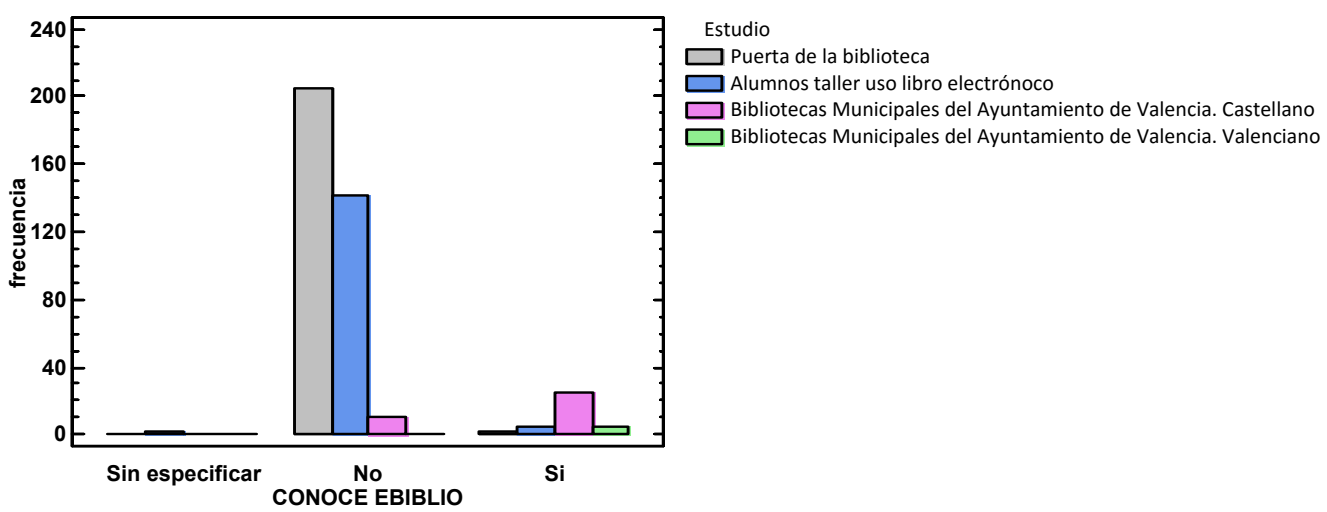

Gráfico 6. Conocimiento servicio EBiblio según estudio. Fuente: Elaboración propia

En la Tabla 3, se han calculado los porcentajes del conocimiento del servicio EBiblio condicionados al estudio (en negrita). Se observa que en los dos primeros estudios estos porcentajes son similares, sin embargo, en los dos últimos son distintos y esto es debido a que las encuestas realizadas en la red de Bibliotecas Municipales de Valencia fueron respondidas principalmente por el propio personal de la biblioteca. En cualquier caso, el número de encuestas en estos dos estudios es mucho mejor que el de los dos anteriores.

\begin{tabular}{|c|c|c|c|c|c|}
\hline \multirow[b]{2}{*}{$\begin{array}{l}\text { Conocimiento } \\
\text { EBiblio }\end{array}$} & \multicolumn{4}{|l|}{ Estudio } & \\
\hline & $\left|\begin{array}{ll}\text { Puerta } & \text { de } \\
\text { Biblioteca } & \end{array}\right|$ & $\begin{array}{ll}\text { Alumnos } & \text { Taller } \\
\text { uso } & \text { Libro } \\
\text { Electrónico }\end{array}$ & $\begin{array}{l}\text { Bibliotecas } \\
\text { Municipales } \\
\text { Ayuntamiento } \\
\text { Valencia. } \\
\text { Castellano }\end{array}$ & $\begin{array}{l}\text { Bibliotecas } \\
\text { Municipales } \\
\text { Ayuntamiento } \\
\text { Valencia. } \\
\text { Valenciano }\end{array}$ & $\begin{array}{l}\text { Total por } \\
\text { Fila }\end{array}$ \\
\hline Sin especificar & 0 & 2 & 1 & 0 & 3 \\
\hline
\end{tabular}




\begin{tabular}{|l|l|l|l|l|l|}
\hline & $\mathbf{0 . 0 0} \%$ & $\mathbf{1 . 3 6} \%$ & $\mathbf{2 . 7 8 \%}$ & $\mathbf{0 . 0 0} \%$ & \\
\hline No & 205 & 141 & 10 & 1 & 357 \\
\hline & $\mathbf{9 9 . 0 3 \%}$ & $\mathbf{9 5 . 9 2} \%$ & $\mathbf{2 7 . 7 8 \%}$ & $\mathbf{1 6 . 6 7 \%}$ & \\
\hline $\mathrm{Si}$ & 2 & 4 & 25 & 5 & 36 \\
\hline & $\mathbf{0 . 9 7 \%}$ & $\mathbf{2 . 7 2} \%$ & $\mathbf{6 9 . 4 4 \%}$ & $\mathbf{8 3 . 3 3 \%}$ & \\
\hline $\begin{array}{l}\text { Total } \\
\text { Columna }\end{array}$ & 207 & 147 & 36 & 6 & 396 \\
\hline & $52.27 \%$ & $37.12 \%$ & $9.09 \%$ & $1.52 \%$ & $100.00 \%$ \\
\hline
\end{tabular}

Tabla 3. Conocimiento servicio EBiblio según el estudio. Fuente: Elaboración propia

En cuanto a la valoración del servicio EBiblio, en el Gráfico 7 se muestra que únicamente el $45.45 \%$ de los encuestados valoran el servicio EBiblio positivamente repartidos el 6.06\% son del estudio realizado en la puerta de la biblioteca, el 3.03\% de los alumnos del taller sobre el uso del libro electrónico y 36.36\% del estudio realizado en las Bibliotecas Municipales de Valencia. Si se tiene en cuenta que en el último estudio la mayor parte de las respuestas correspondían a personal de la biblioteca, la valoración positiva para el servicio EBiblio es significativamente menor.

\section{Satisfacción EBilblio}

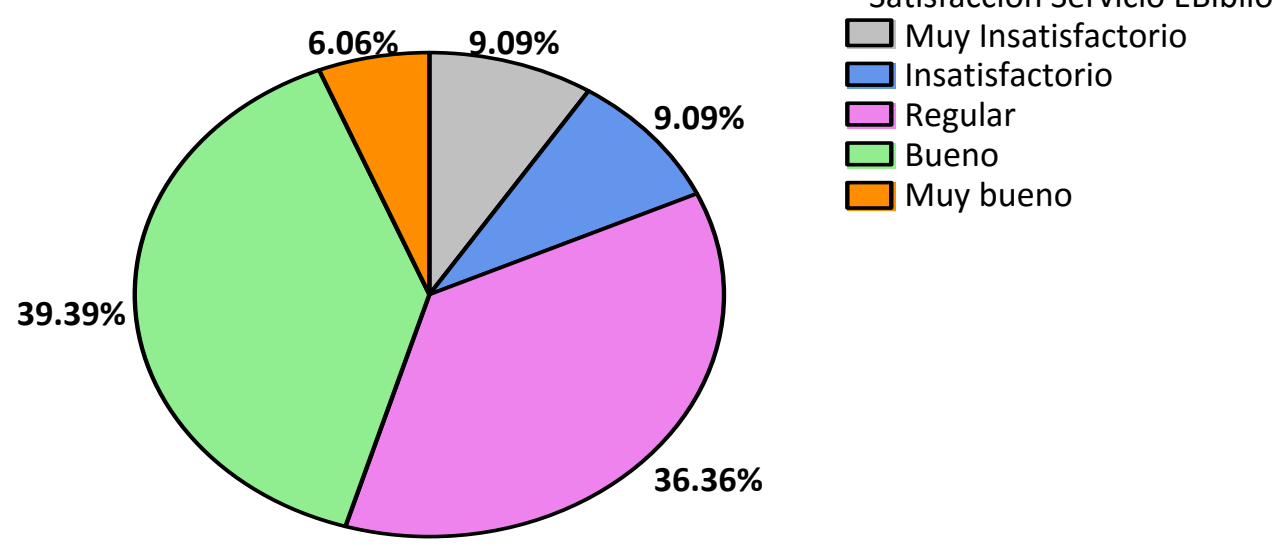

Gráfico 7. Satisfacción servicio EBiblio. Fuente: Elaboración propia

En la Tabla 4 se muestran los factores que dificultan el servicio según la valoración del mismo. Se han calculado los porcentajes sobre el total de respuestas. Cabe destacar que el $46.67 \%$ considera que falta oferta de títulos, el $30 \%$ considera que es difícil el uso del servicio mientras que el $23.33 \%$ considera que hay pocas licencias por título. 


\begin{tabular}{|c|c|c|c|c|c|c|c|}
\hline \multirow[b]{2}{*}{$\begin{array}{l}\text { Factores que dificultan el servicio } \\
\text { EBiblio }\end{array}$} & \multicolumn{5}{|c|}{ Satisfacción Biblioteca } & & \\
\hline & 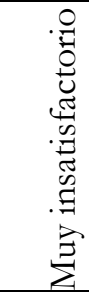 & 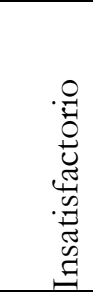 & 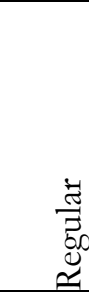 & 节 & 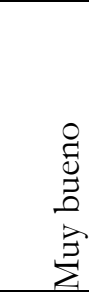 & 丞 & 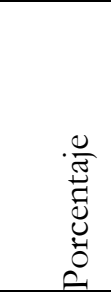 \\
\hline Falta de oferta de títulos & 1 & 0 & 6 & 6 & 1 & 14 & 0.4667 \\
\hline Pocas licencias por título & 0 & 0 & 3 & 4 & 0 & 7 & 0.2333 \\
\hline $\begin{array}{l}\text { Dificultad técnica del uso del } \\
\text { servicio }\end{array}$ & 0 & 1 & 3 & 4 & 1 & 9 & 0.3 \\
\hline $\begin{array}{l}\text { Mi dispositivo actual no es válido } \\
\text { para usar este servicio }\end{array}$ & 1 & 2 & 1 & 1 & 0 & 5 & 0.1667 \\
\hline $\begin{array}{l}\text { No tengo dispositivo, he de } \\
\text { adquirirlo }\end{array}$ & 1 & 0 & 3 & 0 & 0 & 4 & 0.1333 \\
\hline
\end{tabular}

Tabla 4. Factores que dificultad el servicio según la valoración del servicio EBiblio.Fuente:

\section{Elaboración propia}

Respecto a si consideran interesante incluir libros en valenciano, el 96.68\% considera que sería interesante incluirlos.

En la tabla 5 se pregunta por varias afirmaciones sobre el servicio EBiblio para que los encuestados respondan sobre su grado de acuerdo con ellas. Cabe destacar que el $82.35 \%$ consideran que puede servir para luchar contra la piratería. El 91.18\% considera que hay poca información sobre el servicio EBiblio y $80.52 \%$ considera que deberían de estar mejor representados los libros de pequeñas editoriales.

\begin{tabular}{|c|c|c|c|c|c|}
\hline \multirow[b]{2}{*}{$\begin{array}{l}\text { Afirmaciones relativas al servicio } \\
\text { EBiblio }\end{array}$} & \multicolumn{5}{|c|}{ Satisfacción Biblioteca } \\
\hline & 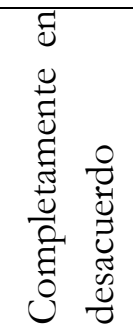 & 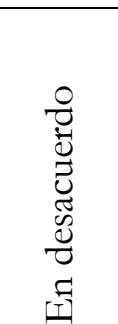 & 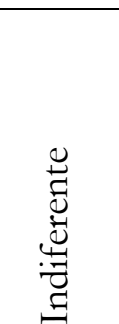 & 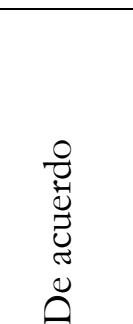 & 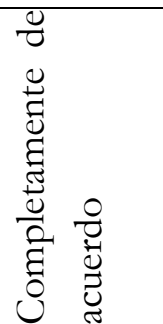 \\
\hline $\begin{array}{l}\text { La plataforma es una buena } \\
\text { oportunidad para luchar contra la } \\
\text { piratería }\end{array}$ & 0.0084 & 0.0042 & 0.1639 & 0.4664 & 0.3571 \\
\hline $\begin{array}{l}\text { Hay poca información sobre } \\
\text { Ebiblio }\end{array}$ & 0 & 0 & 0.0882 & 0.4202 & 0.4916 \\
\hline $\begin{array}{l}\text { Los textos de las pequeñas } \\
\text { editoriales deben estar más } \\
\text { representados por la plataforma }\end{array}$ & 0 & 0 & 0.1907 & 0.4873 & 0.322 \\
\hline
\end{tabular}


Tabla 5. Afirmaciones relativas al servicio EBiblio. Fuente: Elaboración propia Por último, remarcar que al $84.17 \%$ de los encuestados le gustaría recibir información sobre el servicio EBiblio. Esto nos induce a pensar que existe interés por el servicio, pero que habría que darlo a conocer.

\section{Conclusiones}

Una primera conclusión que podemos obtener es que la mayoría de los usuarios de las bibliotecas públicas estudiadas no tiene conocimiento alguno del producto. En el primer estudio, de 207 encuestados, solo 2 conocían el servicio. Un año después, los porcentajes eran casi idénticos.

Seguramente, ello se debe a la falta de visibilidad de este producto en las bibliotecas que siguen muy apegadas a modelos tradicionales de la pasada centuria con el libro en papel como principal protagonista. También a las propias limitaciones de la web actual, del programa http://comunitatvalenciana.ebiblio.es/es/, una página muy estática que carece de ninguna opción de interacción con personas responsables ni por correo electrónico ni mediante contacto teléfono; tampoco está presente en redes sociales. Sería necesario una política activa de difusión del servicio, tanto entre los usuarios de bibliotecas como entre el resto de la población no usuaria de las mismas.

La segunda conclusión es la escasa satisfacción con el producto tal y como hoy se ofrece. Por un lado, existen importantes limitaciones técnicas, pues hoy por hoy solo se permiten su uso por iOs y Android, pero no por el sistema de Amazon, Kindle, el más utilizado en España. En cuanto a los contenidos, la escasa presencia de obras en valenciano o su relación con novedades del mundo editorial, dejan fuera a numerosos posibles lectores que no encontrarán en el escaso catálogo disponible los textos que desean leer. 


\section{Referencias}

ALONSO ARÉVALO, Julio, José Antonio CORDÓN GARCÍA y Raquel GÓMEZ DÍAZ, 2014. Comparación de los hábitos y perfil del lector digital entre Estados Unidos y España. Anales de documentación, 17(1), 16 págs. ISSN-e 1697-7904. CUADRADO FERNÁNDEZ, Ma Isabel y Ma José FE TRILLO, 2015. EBiblio, servicio de préstamo de libros electrónicos en bibliotecas públicas. El profesional de la información, 24(2), 176-184. ISSN 1386-6710.

DOMÍNGUEZ SÁNCHEZ, Pilar, et al., 2011. Desarrollo del servicio de préstamo en línea de libros electrónicos en las bibliotecas públicas de Cataluña y de la Comunidad de Madrid. BiD: textos universitaris de biblioteconomia $i$ documentació, 26 [Consulta: 20-12-2016]. Disponible en: < http://bid.ub.edu/26/vives2.htm>. DOI: $\underline{\text { http://dx.doi.org/10.1344/105.000001725. }}$.

EBLIDA, 2014. El derecho a leer en electrónico. Bruselas: EBLIDA [Consulta: 20-XII2016]. Disponible en: <http://www.eblida.org>.

GÓMEZ-GÓMEZ, Antonio Agustín, 2015. Balance del primer año de funcionamiento de eBiblio Andalucía: el servicio de préstamo de libros electrónicos de las bibliotecas públicas. Boletín de la Asociación Andaluza de Bibliotecarios, 109, 161 171. ISSN 0213-6333.

MINISTERIO DE EDUCACIÓN, CULTURA Y DEPORTE, 2016. EBiblio2015: estadísticas. Madrid: Ministerio de Educación, Cultura y Deporte [Consulta: 20-XII2016]. Disponible en: < $\underline{\text { http://www.mecd.gob.es/cultura- }}$ $\mathrm{mecd} / \mathrm{dms} / \mathrm{mecd} /$ cultura-mecd/areascultura/bibliotecas/eBiblio/eBiblio 2015 est.pdf $>$.

MOUNT, Dan, 2014. A review of public library e-lending models. Brussels: European Union [Consulta: 20-12-2016]. Disponible en: $<$ http://www.bibliotheksportal.de/fileadmin/user upload/content/bibliothe ken/international/Study Public Library E-Lending Models 2014.pdf>.

OBSERVATORIO DE LA LECTURA Y EL LIBRO, 2016. El sector del libro en España 2013-2015. Madrid: Ministerio de Educación, Cultura y Deporte [Consulta: 20-XII-2016]. Disponible en: < http://www.mecd.gob.es>. 
PÉREZ LÓPEZ, Sara, 2015. Ebiblio Comunidad Valenciana. Conocimiento y satisfacción de usuarios. Valencia: Universitat Politècnica de València. Trabajo final de la Diplomatura en Gestión y Administración Pública, dirigido por Nuria Portillo Poblador y Miguel C. Muñoz Feliu.

Vicente García, Remedios de, 2013. Los libros electrónicos en las colecciones de las bibliotecas públicas. BiD: textos universitaris de biblioteconomia $i$ documentació, 30 [Consulta: 20-12-2016]. Disponible en: < http://bid.ub.edu/es/30/devicente.htm $>$. DOI: http://dx.doi.org/10.1344/BiD2013.30.11.

\section{AGRADECIMIENTOS}

Este estudio no hubiera sido posible sin la colaboración de Sara Pérez y el apoyo de Marga Valls y del Ajuntament de València. 